\title{
Non-nilpotent Subgroups in Locally Graded Groups
}

\author{
N. Azimi, M. Amirabadi* \\ Department of Mathematics, Hamedan branch, Islamic azad University, Hamedan, Iran \\ *Corresponding Author: Amirabadi@iauh.ac.ir
}

Copyright (C)2014 Horizon Research Publishing All rights reserved.

\begin{abstract}
A non-nilpotent finite group whose proper subgroups are all nilpotent (or a finite group without nonnilpotent proper subgroups) is well-known (called Schmidt group). O.Yu. Schmidt (1924) studied such groups and proved that such groups are solvable. More recently Zarrin generalized Schmidt's Theorem and proved that every finite group with less than 22 non-nilpotent subgroups is solvable. In this paper, we show that every locally graded group with less than 22 non-nilpotent subgroups is solvable.
\end{abstract}

Keywords Schmidt Group, Locally Graded Group, Solvable Group

\section{Mathematics Subject Classification 20 D99}

(2000)

\section{Introduction and Results}

Let $G$ be a group. A non-nilpotent finite group whose proper subgroups are all nilpotent (or a finite group without non-nilpotent proper subgroups) is well-known (called Schmidt group). O.Yu. Schmidt (1924) studied such groups and proved that such groups are solvable [4]. Subsequently, Newman and Wiegold in [1], discuss infinite non-nilpotent groups whose proper subgroups are all nilpotent. Such groups need not be solvable in general. For example, the Tarski Monsters, which are infinite simple groups with all proper subgroups of a fixed prime order. Following [7] we say that a group $G$ is a $\mathcal{S}^{n}$-group if $G$ has exactly $n$ nonnilpotent subgroups. Also we say that $G$ has a finite number of non-nilpotent subgroups, written $G \in \mathcal{S}$, if $G \in \mathcal{S}^{n}$ for some $n \in \mathbb{N}$. Indeed $\mathcal{S}=\bigcup_{i>1} \mathcal{S}^{i}$. The $\mathcal{S}^{n}$-groups $G$ with $n=1$, namely non-nilpotent groups all of whose proper subgroups are nilpotent (i.e. minimal non-nilpotent groups). Therefore Schmidt's Theorem says that every finite $\mathcal{S}^{1}$-group is solvable. More recently Zarrin in [7] generalized Schmidt's Theorem and proved that every finite $\mathcal{S}^{n}$-group with $n<22$ is solvable. But this result is not true for infinite $\mathcal{S}^{n}$-groups (for instance the Tarski Monsters groups are infinite $\mathcal{S}^{1}$-groups).

Here we improve this result as follows:

Theorem 1.1. Every locally graded (or, locally solvable) $\mathcal{S}^{n}$-group with $n<22$ is solvable.
A group $G$ is said to be locally graded if every non-trivial finitely generated subgroup of $G$ has a non-trivial finite homomorphic image. This is a rather large class of groups, containing for instance all residually finite groups and all locally(soluble by finite) groups. It is often considered in order to avoid Tarski monsters groups.

A non-abelian finite group whose proper subgroups are all abelian is well-known (called Miller-Moreno group). In 1903, Miller and Moreno pioneered the study of such groups and proved that such groups are solvable [3]. Now, using the above result and as every non-nilpotent subgroup is a non-abelian subgroup, we generalize their result as follows.

Corollary 1.2. Every locally graded (or, locally solvable) group with less than 22 non-abelian subgroups is solvable.

\section{Proof}

For the proof of main Theorem, we using similar methods as [8]. It is well known that if $G$ is a group and $H$ is a normal subgroup of $G$ such that $H$ is solvable and $G / H$ is solvable then $G$ is solvable

Proof of Theorem 1.1. Suppose that $G$ is a $\mathcal{S}^{n}$-group with $n \leq 21$. Put $X=\{H \mid$ $H$ is a non-nilpotent subgroup of $G\}$. The group $G$ acts on the set $X$ by conjugation. Now the subgroup

$$
A N(G)=\bigcap_{H \in X} N_{G}(H)
$$

is the kernel of this action, so $A N(G)$ is normal in $G$ and $G / A N(G)$ embeds in the symmetric group of degree $n$, where $N_{G}(H)$ is the normalizer $H$ in $G$. Therefore $\frac{G}{A N(G)}$ is finite and also, by Lemma 2.3 of [7], $\frac{G}{A N(G)}$ is a $\mathcal{S}^{r}$-group with $r<22$. Therefore, according to the main result of [7], to complete the proof it is enough to show that $A N(G)$ is solvable. To see this, suppose that $K$ is a non-nilpotent of $A N(G)$. It follows that $K$ is a non-nilpotent of $G$ and so, by definition of $A N(G)$, we obtain that $K \triangleleft A N(G)$. Therefore every non-nilpotent subgroup of $A N(G)$ is normal (and hence is subnormal).

If $G$ is a locally graded group, then $A N(G)$ is also locally graded group and so, by Theorem 2 of [5], it follows that $A N(G)$ is solvable.

If $G$ is a locally solvable group, then $A N(G)$ is also locally 
solvable group and so, by the main result of [2], $A N(G)$ either is locally nilpotent or has finite commutator subgroup. Now if $A N(G)$ is locally nilpotent, then it follows from Lemma 2.2 of [5], that $A N(G)$ is a solvable group. Finally, if $A N(G)^{\prime}$ the commutator subgroup of $A N(G)$ is finite then the main result of [7] follows that $A N(G)^{\prime}$ is a solvable and so $A N(G)$ is solvable and this completes the proof.

Remark 2.1. In view of the proof of the Theorem, we can see that every locally graded (or, locally solvable) $\mathcal{S}$-group is solvable-by-finite. More precisely, if $G$ is an arbitrary group with a finite number $n$ of non-nilpotent subgroups, then the factor group $\frac{G}{A N(G)}$ is finite and $\left|\frac{G}{A N(G)}\right| \leq n$ !. This result suggests that the behaviour of non-nilpotent subgroups has a strong influence on the structure of the group.

Smith in [6] showed that every torsion-free locally solvable-by-finite group in which every non-nilpotent subgroup is subnormal is nilpotent. Now by a similar argument as in the proof of the Theorem, mentioned for $A N(G)$, we can give the following Corollary.

Corollary 2.2. Every torsion-free locally solvable-by-finite group with a finite number of non-nilpotent subgroups is nilpotent-by-finite.

Proof. Assume that $G$ is a torsion-free locally solvable-byfinite group with a finite number of non-nilpotent subgroups. Then, according to Remark 2.1, the factor group $\frac{G}{A N(G)}$ is finite and the subgroup $A N(G)$ is solvable since every locally solvable-by-finite group is locally graded group. On the other hand, $A N(G)$ is torsion-free locally solvable-by-finite group and also, as we mentioned in the proof of main Theorem, every non-nilpotent subgroup of $A N(G)$ is normal. There- fore, by [6], one can follow that $A N(G)$ is nilpotent and this completes the proof.

\section{Acknowledgements}

I would like to thank the referee for useful comments.

\section{REFERENCES}

[1] M.F. Newman and J. Wiegold, Groups with many nilpotent subgroups, Archiv der Math. 15 (1964), 241-250.

[2] B. Bruno and R. E. Phillips, Groups with restricted nonnormal subgroups, Math.Z. 176 (1981), 199-221.

[3] G.A. Miller and H. C. Moreno. Non-abelian groups in which every subgroup is abelian. Trans. Amer. Math. Soc. 4 (1903), 398-404.

[4] O. Yu. Schmidt, Groups all of whose subgroups are nilpotent, Mat. Sbornik 31 (1924), 366-372. (Russian).

[5] H. Smith, Groups with all non-nilpotent subgroups subnormal, Quaderni di Matematica (Caserta) 8 (2001), 309-326.

[6] H. Smith, Torsion-free groups with all non-nilpotent subgroups subnormal, Quaderni di Matematica (Caserta) 8 (2001), 297-308.

[7] M. Zarrin, A generalization of Schmidt's Theorem on groups with all subgroups nilpotent, Arch. Math. (Basel) 99 (2012), 201-206.

[8] M. Zarrin, Non-subnormal subgroups of groups, Journal of Pure and Applied Algebra 217 (2013) 851-853. 